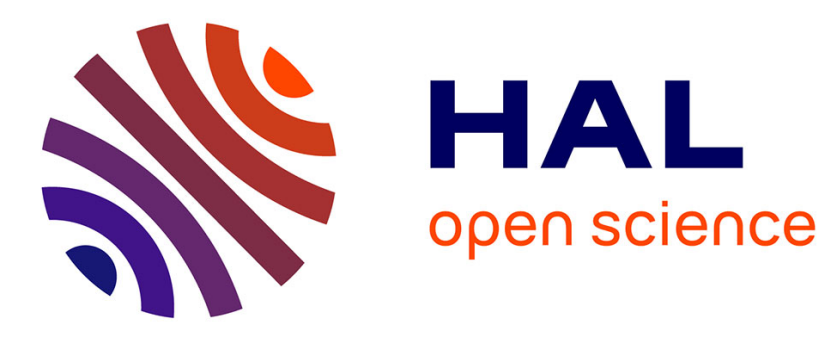

\title{
Parity violation in nuclear systems
}

\author{
B. Desplanques
}

\section{To cite this version:}

B. Desplanques. Parity violation in nuclear systems. PAVI04 - International Workshop on Parity Violation and Hadronic Structure, Jun 2004, Grenoble, France. pp.171-174, 10.1140/epjad/s2005-04043-9 . in2p3-00023522

\section{HAL Id: in2p3-00023522 https://hal.in2p3.fr/in2p3-00023522}

Submitted on 5 Jan 2005

HAL is a multi-disciplinary open access archive for the deposit and dissemination of scientific research documents, whether they are published or not. The documents may come from teaching and research institutions in France or abroad, or from public or private research centers.
L'archive ouverte pluridisciplinaire HAL, est destinée au dépôt et à la diffusion de documents scientifiques de niveau recherche, publiés ou non, émanant des établissements d'enseignement et de recherche français ou étrangers, des laboratoires publics ou privés. 


\title{
Parity violation in nuclear systems
}

\author{
B. Desplanques \\ Laboratoire de Physique Subatomique et de Cosmologie (UMR CNRS/IN2P3-UJF-INPG), \\ F-38026 Grenoble Cedex, France
}

Received: date / Revised version: date

\begin{abstract}
Parity violation in nuclear systems is reviewed. A few ingredients relevant to the description of the parity-violating nucleon-nucleon force in terms of meson exchanges are reminded. Effects in nuclear systems are then considered. They involve $p p$ scattering, some complex nuclei and the deuteron system.
\end{abstract}

PACS. 24.80.+y Nuclear tests of fundamental interactions and symmetries

\section{Introduction}

A large number of parity-non-conserving (pnc) effects has been observed in various nuclear systems. While their ex$\curvearrowleft$ pected size at low energy is of the order of $10^{-7}$ (for the , amplitude), they can be strongly enhanced in some cases, due to the closeness of states with opposite parities or the

suppression of the regular transition. Thus, effects of the F order of $10^{-1}$ have been measured in neutron-nucleus scattering in the vicinity of low energy p-wave resonances (see $\checkmark$ ref. [1] for some review). Qualitatively, such effects are understood. However, little quantitative information could $>$ be obtained on the pnc component of nucleon-nucleon $(N N)$ forces expected to account for them. From the know$\checkmark$ ledge of this interaction, one can expect to learn about the $\checkmark$ pnc meson-nucleon coupling constants which they depend on and, thus, get information on the underlying hadronic mphysics. This one is complementary to the information that can be obtained from non-leptonic hyperon decays. It concerns in first place the $\pi N N$ coupling that has been at the center of many theoretical and experimental works. I This one can be most easily compared to non-leptonic hy(3) peron decay amplitudes. Another less fundamental but im$=$ portant motivation for the study of pnc nuclear effects is $\checkmark$ the necessity to determine the effective strength of the various pnc $N N$ amplitudes. These ones can indirectly contribute to other pnc effects, especially in electron scat(1) tering mainly discussed at this meeting. Though the effect

$\checkmark$ is not large, its knowledge is required to determine the reliability of the information that is looked for in such high accuracy measurements. Some recent developments in the field are reviewed here.

The plan of the paper is as follows. In the second part, we briefly remind ingredients entering the pnc $N N$ force, while emphasizing a few points of interest for the following part devoted to pnc effects. The third section is concerned with pnc $p p$ scattering. This process is the only one that provides a calibration of the strength of pnc $N N$ forces at the present time. A few nuclear pnc effects in complex nuclei, especially in ${ }^{18} \mathrm{~F}$ and in ${ }^{133} \mathrm{Cs}$, are discussed in the fourth section. The fifth section is devoted to pnc effects in the $n p$ system, including the deuteron. This particular field has been particularly active these last years. A conclusion and an outlook are presented in the sixth section.

\section{PNC $N N$ potential: ingredients}

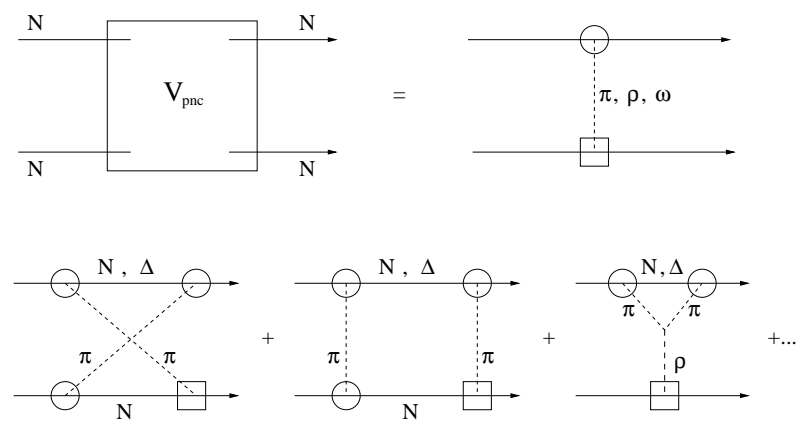

Fig. 1. Diagram representation of the pnc $N N$ interaction.

The pnc $N N$ force is generally described as resulting from meson exchanges, $\pi, \rho$ and $\omega$. A diagrammatic representation is given in fig. 1. One of the vertex, represented by a circle, corresponds to the strong interaction. The other one, represented by a box, corresponds to the weak, pnc, interaction. As isospin is not conserved, there are many couplings in some cases. They are:

- $h_{\pi}^{1}$, which governs the long range part of the force and necessarily involves the $\Delta T=1$ component of the weak interaction

- $h_{\rho}^{0,1,2}: \Delta T=0,1,2$

- $h_{\omega}^{0,1}: \Delta T=0,1$

- $h_{\rho}^{1^{\prime}}: \Delta T=1$, (different type $\rho N N$ coupling). 
The vector-meson couplings determine the short-range part of the pnc $N N$ force. As is well known, the contribution of this part is sensitive to short-range correlations in the strong $N N$ interaction as well as to other correlations.

Many contributions going beyond the above ones have been considered in the literature. They involve for instance two-pion exchanges displayed in fig. 1, either with the same coupling as for the one-pion exchange or with the same coupling as for the $\rho$ exchange. In the last case, the $\rho$-exchange force acquires a longer range that could show up in the analysis of pnc effects in $p p$ scattering. Some discussion and references could be found in ref. [1].

At low energy, only gross features may be relevant. The $N N$ interaction can then be parametrized by five $S \leftrightarrow P$ $N N$ transition amplitudes [2]:

${ }^{1} S_{0} \leftrightarrow{ }^{3} P_{0}, \Delta T=0,1,2,(p p, p n$ and $n n$ forces $)$

$-{ }^{3} S_{1} \leftrightarrow{ }^{1} P_{1}, \Delta T=0$ (pn force)

- ${ }^{3} S_{1} \leftrightarrow{ }^{3} P_{1}, \Delta T=1$ (pn force).

It was shown that this description could be extended to higher energy by singularizing the pion-exchange contribution which, due to its long range, contributes sizeable $P \leftrightarrow D$ transition amplitudes [3]. Apart from the name, these works largely anticipated recent effective field-theory approaches [4, which also consider $P \leftrightarrow D$ transitions.

Many works have been devoted to the pnc mesonnucleon couplings, which enter $N N$ interaction models. A large part of them, prior to the DDH work [5] or later, fit in this framework. Due to the lack of space, we again refer to ref. [1] for references and detailed discussion. We only present here some estimates and make a few pertinent remarks. The sample of results given in table 1 corresponds to the predictions of two significantly different models for the most relevant couplings, $h_{\pi}^{1}, h_{\rho}^{0}$ and $h_{\omega}^{0}$. They are based on a quark model (DDH), partly updated, and a chiral soliton model by Kaiser and Meissner (KM) [6] (see also ref. [7]). Despite appearances, results turn out to be qualitatively similar. Discrepancies can be ascribed to the weight attributed to individual contributions in DDH. It is noticed that the dominant contribution to $h_{\pi}^{1}$ is produced by strange quarks, of particular interest at this meeting while the consistency of this estimate with the QCD sum rules ones remains an open problem. It was proposed to use a chiral quark model to make a new estimate (Lee et al. 8], this conference). It is also noticed that $\mathrm{DDH}$ estimates, relying for a part on experimental data, should be less sensitive to "rescattering effects" evoked in the literature [9] whereas $h_{\omega}^{0}$ is likely to be negative.

\section{Longitudinal asymmetry in $p p$ scattering}

The low-energy longitudinal asymmetry in $p p$ scatering is the most important benchmark in the field at present. A complete theoretical analysis can be done. It shows that measurements at 13.6 and $45 \mathrm{MeV}$ are in complete agreement with each other, thus fixing the strength of the ${ }^{1} S_{0} \leftrightarrow{ }^{3} P_{0}$ pnc $p p$ transition amplitude. For a given description of the strong interaction model, the strength of a combination of $h_{\rho}^{p p}$ and $h_{\omega}^{p p}$ couplings or, in first approximation, the $h_{\rho}^{0}$ and $h_{\omega}^{0}$ couplings, can be obtained. At
Table 1. Meson-nucleon pnc coupling constants: a few estimates from different works. The question mark at the last line indicates that the original value could be actually close to 0 .

\begin{tabular}{cccc}
\hline & DDH (range) & DDH("best" ) & KM \\
\hline $10^{7} h_{\pi}^{1}$ & $0 \leftrightarrow 11$ & 4.6 & 0.2 \\
$10^{7} h_{\pi}^{1}$ & $0 \leftrightarrow 2.5$ & & $0.8-1.3$ \\
& (update, $K=3$ ) & & (with $\bar{s} s)$ \\
$10^{7} h_{\rho}^{0}$ & $-31 \leftrightarrow 6$ & -11 & -4 \\
$10^{7} h_{\omega}^{0}$ & $-10 \leftrightarrow 6 ?$ & -2 & -6 \\
\hline
\end{tabular}

higher energy, around $221 \mathrm{MeV}$, it was noticed that the contribution of the ${ }^{1} S_{0} \leftrightarrow{ }^{3} P_{0}$ transition amplitude was vanishing, providing a window to determine the ${ }^{3} P_{2} \leftrightarrow$ ${ }^{1} D_{2}$ transition amplitude. By combining this measurement with the low-energy one, contributions due to $\rho$ and $\omega$ exchanges can thus be disentangled.

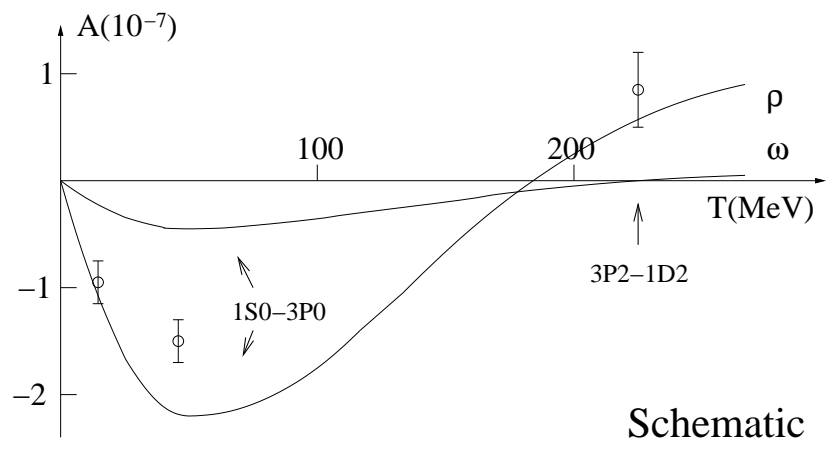

Fig. 2. Schematic representation of $\rho$ - and $\omega$-exchange contributions to the longitudinal asymmetry in $p p$ scattering.

A schematic representation of the two contributions with couplings close to the DDH "best guess" ones, is given in fig. 2. It is seen that the $\omega$-exchange one has a negligible contribution around $221 \mathrm{MeV}$ while a $\rho$-exchange contribution alone is not doing badly. A better agreement is obtained by increasing the strength of this one and compensating for the overestimate at low energy by adding a $\omega$-exchange contribution with a sign opposite to the DDH "best guess" or KM one. This provides a simple explanation for the couplings obtained by Carlson et al. [10:

$$
\begin{aligned}
& h_{\rho}^{p p}=-22.310^{-7}, \quad h_{\omega}^{p p}=+5.210^{-7} \quad \text { (fit) } \\
& h_{\rho}^{p p}=-15.510^{-7}, \quad h_{\omega}^{p p}=-3.010^{-7}, \quad \text { (DDH). }
\end{aligned}
$$

The fit evidences a striking feature as the value for the $\omega$ coupling has a sign opposite to expectations and, thus, can point to missing ingredients in predictions. We however notice that the significance of the result is not strong (the $\rho$ alone is already giving a good account). A refined theoretical analysis and a more accurate measurement could be quite useful. It would be interesting to investigate for instance the role of a longer range $\rho$-exchange contribution mentioned in the second section. 


\section{Parity-non-conservation in complex nuclei}

Many pnc effects in complex nuclei have been measured and analyzed. Considered individually, it is however difficult to draw some conclusion from them. Moreover, when they are considered together, it is not rare that the information obtained from one process is at the limit to contradict that one from another process. Two effects nevertheless deserve some attention: the circular polarization of photons emitted in the transition $0^{-}(1.08 \mathrm{MeV}) \rightarrow$ $1^{+}$(g.s.) in ${ }^{18} \mathrm{~F}$ and the ${ }^{133} \mathrm{Cs}$ anapole moment. They are successively discussed in the following (references for both theory and experiment may be found in ref. [1]).

The interest of the pnc effect in ${ }^{18} \mathrm{~F}$ is that the calculation of the relevant pnc nuclear matrix element $\left(0^{-} \leftrightarrow 0^{+}\right)$ can be checked by looking at the first forbiden $\beta$ decay of the neighboring nucleus, ${ }^{18} \mathrm{Ne}$. It implies the $\Delta T=1$ part of the weak interaction and the measurement can thus provide information on the pnc $\pi N N$ coupling, $h_{\pi}^{1}$. From the experimental limit of $P_{\gamma}$, one gets the following upper limit:

$$
\left|h_{\pi}^{1}\right| \leq 1.310^{-7} .
$$

This result is supported by the absence of effect in two other processes in ${ }^{21} \mathrm{Ne}$ and in ${ }^{93} \mathrm{Tc}$. In these cases, the contribution of the pion exchange is a priori large. To agree with the upper experimental limit, one has first to assume that the coupling $h_{\pi}^{1}$ is not too large and, moreover, that the corresponding contribution be cancelled for a part by some isoscalar contribution (for ${ }^{21} \mathrm{Ne}$ ). Contrary to ${ }^{18} \mathrm{~F}$, there is no available check on the relevant pnc nuclear matrix element. Accepting that this one be uncertain by up to a factor 3 would however give a limit on $h_{\pi}^{1}$ similar to eq. (2).

The ${ }^{133} \mathrm{Cs}$ anapole moment has been analyzed by different authors. To a large extent, this quantity involves a combination of the pnc $N N$ force close to that one governing pnc effects in several odd-proton systems as different as $p \alpha$ scattering, ${ }^{19} \mathrm{~F},{ }^{41} \mathrm{~K},{ }^{175} \mathrm{Lu},{ }^{181} \mathrm{Ta}$. At first sight, it appears that the above combination should be two times larger for the anapole moment than for the other processes. The discrepancy has the order of a typical uncertainty in the field but there was some belief that the estimate in the first case could be less uncertain than for other effects (for a part, it involves a long-range operator). On the other hand, the effects in the other odd-proton systems overdetermine the above combination of parameters. If these last processes are ignored, it appears that a large value of $h_{\pi}^{1}$, of the order of $110^{-6}$, at the upper limit of the original DDH range, is needed. This is inconsistent both with the upper limit, eq. (2), and the DDH updated range. We notice that the last calculation of the anapole moment 11 relies on an approximation that allows for an improved calculation in one respect but implies some contribution from orbitals below the Fermi level with a wrong sign in another respect. A correct account of these ones could enhance the theoretical estimate but will not reach a factor 2 . The validity of a similar approximation, which omits 3-body terms, was discussed in ref. [12].

\section{Parity-non-conservation in the deuteron}

Most recent pnc studies in nuclear systems have concentrated on the $n p$ system (deuteron and scattering state). This emphasis is largely motivated by both the feasibility of the corresponding experiments in a near future (see Stiliaris's talk at this conference) and a safer interpretability of possible effects. These ones include the photon-emission asymmetry in the thermal-energy radiative capture of polarized neutrons by protons, $\boldsymbol{n}+p \rightarrow d+\gamma$ [13, 14, 15, 16], presently performed at LANSCE, the asymmetry in the deuteron photo-disintegration depending on the photon helicity [17, 18, 19, 20], which could be performed at JLab, IASA, SPring-8, $\cdots$, the deuteron anapole moment [21, 22, 23,24, the pnc deuteron electro-disintegration in relation with the SAMPLE experiment [25, 16], and the longitudinal asymmetry and the neutron-spin rotation in $n p$ scattering [20]. Some earlier works could be quoted. The recent ones involve new methods (effective-field theories [13. 15), improved $N N$ interaction models (AV18+ $\cdots$ [15, 24, 16, 18, 20), more complete calculations (two-body currents [25. 16]), and increased attention to gauge invariance [23. 24.20]. A few remarks are made below about these different works.

The earlier pion-exchange contribution to the asymmetry in the thermal-neutron radiative capture on protons, $\boldsymbol{n}+p \rightarrow d+\gamma$,

$$
A_{\gamma}=-0.11 h_{\pi}^{1},
$$

is confirmed by recent estimates, indicating that the correction for a wrong ${ }^{1} S_{0} n p$ scattering length, was appropriately made. It is also found that the above estimate results from a strong cancellation when a calculation is performed without relying on the Siegert theorem [15, 16]. Amazingly, this weak interaction problem provides information on the accuracy of effective-field theory methods employed for the strong interaction. The approach used in ref. [13], for instance, overestimes eq. (3) by $60 \%$ at leading order (almost a factor 2 for comparable ingredients).

On the basis of an estimate by Oka 26, it was thought that the study of the photon-helicity dependence of the deuteron photo-disintegration cross section could provide an alternative way to determine the coupling $h_{\pi}^{1}$. This motivated several works that disproved the above estimate and its main conclusion [17, 18, 19, 20]. An account of the new results can be found in the Hyun's talk at this conference. For the inverse process near threshold ("Lobashov experiment"), it should be noticed that a circular polarization of photons as large as $110^{-7}$ is not excluded for some reasonable models of both the strong and the weak $N N$ interaction [20].

The deuteron anapole moment is largely academic as there is not much hope it could be measured in a near future. It however provides a nice laboratory for studying the implications of gauge invariance, which is essential for getting a consistent estimate of this quantity. A contribution required by chiral symmetry [21], absent in ref. [22], has thus been recovered in potential based approaches [23]. On the other hand, this last work confirms the conclusion obtained from the study of $A_{\gamma}$ about the 
reliability of some effective-field theories. It is likely that an alternative approach 27], which is nothing but the one initiated by Danilov's work [2], extended later on to higher energy [3], should do better.

In pnc-electron experiments performed on the deuteron, aiming at determining the contribution of strange quarks to nucleon form factors, there was some concern about the role of a nuclear pnc effect. This one was studied in two different works 25, 16] which showed that the effect, a few percents, would be negligible. Actually, the main role of pnc nuclear effects in this process (together with that one involving the proton) is an indirect one. They allow one to put limits on coupling constants that enter radiative corrections 28].

Parity-non-conservation in $n p$ scattering has been recently revisited [20]. The main feature evidenced by the new results is the dominance of the pion-exchange contribution, as far as the DDH "best guess" is used for the corresponding pnc coupling.

\section{Discussion and conclusion}

Many low-energy pnc nuclear effects, involving mainly protons, are within expectations. However, one has often to be satisfied with discrepancies up to a factor 2. This is not enough to constrain the different pnc meson-nucleon couplings if one refers to a potential approach or the lowenergy $N N$ scattering amplitudes if one rather relies on the less ambitious approach represented by effective field theories.

Most probably, the pnc $\pi N N$ coupling, $h_{\pi}^{1}$, is small and within the DDH updated range. Some processes could require a significantly larger value but, in our opinion, they have not the weight of the other ones that point to a small value. Concerning the vector meson-nucleon couplings, there is a slight hint that the isoscalar $\omega$ one, $h_{\omega}^{0}$, could have a sign opposite to expectations. This should motivate further studies to confirm the hint on the one hand, to see whether this opposite sign is conceivable.

An analysis in terms of couplings has some interest but, apart from the fact it assumes that multi-meson exchanges can be ignored, it does not necessarily provide a pertinent clue at which part of the pnc interaction is rather unconstrained. Looking at the various $N N$ scattering amplitudes can thus represent a complementary view. Among the five amplitudes required for the description of pnc effects at low energy, only one $(p p)$ is determined with a good accuracy. From the study of odd-proton systems, and after removing the contribution of the $p p$ amplitude, a pn amplitude involving "unpolarized" neutrons can be obtained. Being derived indirectly, from complex systems moreover, the accuracy of this amplitude is not as good as for the $p p$ one. For the three other amplitudes, which involve "polarized" neutrons (with "unpolarized" protons, with "unpolarized" neutrons and with "polarized" protons), only upper limits are known.

To determine this sector of the pnc $N N$ interaction, appropriate experiments are heavily needed, preferentially with light systems where theoretical uncertainties are reduced. The $n p$ amplitude with "polarized" neutrons is better studied in the neutron-spin rotation. The $n n$ amplitude could be obtained from the neutron-spin rotation in neutron- $\alpha$ scattering, after removing the previous contribution of the $n p$ amplitude. The best process for determining the last $n p$ amplitude, which involves both "polarized" neutrons and "polarized" protons, is the circular polarization of photons in the thermal neutron-proton radiative capture ("Lobashov experiment"). Evidently, the asymmetry $A_{\gamma}$, already mentioned, is part of the needed experiments. While it involves the difference in the two $n p$ amplitudes with a "polarized" neutron and an "unpolarized" proton on the one hand, the inverse configuration on the other hand, it also allows one to get information on the most debated pnc coupling, $h_{\pi}^{1}$.

\section{Acknowledgments}

We are very grateful to C. H. Hyun and C.-P. Liu for a stimulating and fruitful collaboration.

\section{References}

1. B. Desplanques, Phys. Rep. 297 (1998) 1.

2. G. S. Danilov, Sov. J. Nucl. Phys. 14 (1972) 443; Phys. Lett. 18 (1965) 40; Phys. Lett. B 35 (1971) 579.

3. B. Desplanques and J. Missimer, Nucl. Phys. A 300 (1978) 286.

4. B. Holstein et al., nucl-th/0407087

5. B. Desplanques, J. Donoghue and B. Holstein, Ann. of Phys. 124 (1980) 449.

6. N. Kaiser and U. G. Meissner, Nucl. Phys. A 489 (1988) 671, A 499 (1989) 69, A 510 (1990) 759.

7. U. G. Meissner and H. Weigel, Phys. Lett. B 447 (1999) 1.

8. H.-J. Lee et al., hep-ph/0405217.

9. S.-L. Zhu et al., Phys. Rev. D 63 (2001) 033006.

10. J. Carlson et al., Phys. Rev. C 65 (2002) 035502.

11. W.C. Haxton et al., Phys. Rev. C 65 (2002) 045502.

12. B. Desplanques, Phys. Lett. B 47 (1973) 212.

13. D.B. Kaplan et al., Phys. Lett. B 449 (1999) 1.

14. B. Desplanques, Phys. Lett. B 512 (2001) 305.

15. C. H. Hyun et al., Phys. Lett. B 516 (2001) 321.

16. R. Schiavilla et al., Phys. Rev. C 67 (2003) 032501R.

17. I.B. Khriplovich et al., Nucl. Phys. A 690 (2001) 610.

18. C.-P. Liu, C.H. Hyun and B. Desplanques, Phys. Rev. C 69 (2004) 065502.

19. M. Fujiwara et al., Phys. Rev. C 69 (2004) 065503.

20. R. Schiavilla, J. Carlson and M. Paris, nucl-th/0404082.

21. M.J. Savage and R.P. Springer, Nucl. Phys. A 644 (1998) 235; A 657 (1999) 457(E).

22. I. B. Khriplovich et al., Nucl. Phys. A 665 (2000) 365.

23. C.H. Hyun and B. Desplanques, Phys. Lett. B 552 (2003) 41.

24. C.-P. Liu, C.H. Hyun and B. Desplanques, Phys. Rev. C 68 (2003) 045501.

25. C.-P. Liu et al., Phys. Rev. C 67 (2003) 035501.

26. T. Oka, Phys. Rev. D 27 (1983) 523.

27. M.J. Savage, Nucl. Phys. A 695 (2001) 365.

28. S.-L. Zhu et al., Phys. Rev. D 62 (2000) 033008. 